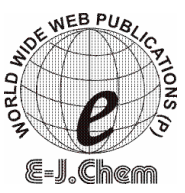

http://www.e-journals.net
ISSN: 0973-4945; CODEN ECJHAO

E-Journal of Chemistry

Vol. 5, No.1, pp. 23-33, January 2008

\title{
Synthesis and Antimicrobial Activity of Some Novel Substituted Piperazinyl-quinazolin-3(4H)-ones
}

\author{
N. M. RAGHAVENDRA*, P. P. THAMPI \\ and P.M. GURUBASAVARAJASWAMY \\ Medicinal Chemistry Research Laboratory, \\ Acharya and B.M. Reddy College of Pharmacy, \\ Bangalore, Karnataka, India \\ nmraghu76@yahoo.co.in
}

Received 26 July 2007; Accepted 20 September 2007

\begin{abstract}
Several substituted-quinazolin-3(4H)-ones were synthesized by condensation of 2-chloro- $N$-(4-oxo-substituted-quinazolin-3(4H)-yl)-acetamides with various substituted piperazines through single step reaction. Elemental analysis, IR, ${ }^{1} \mathrm{HNMR}$ and mass spectral data confirmed the structure of the newly synthesized compounds. Synthesized quinazolin-4-one derivatives were investigated for their antibacterial and antifungal activities.
\end{abstract}

Keywords: Quinazolin-4-ones, Piperazinyl quinazolin-4-ones, Antibacterial activity, Antifungal activity.

\section{Introduction}

Pharmacologically, quinazolin-4-ones are among the most important classes of heterocyclic compounds. These compounds possess versatile type of biological activities; some of these are well known for their anticancer ${ }^{1-2}$, antitubercular ${ }^{3}$, antibacterial ${ }^{4}$, antifungal ${ }^{5}$, anti-HIV ${ }^{6}$, anthelmintic ${ }^{7}$, anti-inflammatory ${ }^{8}$ and antihypertensive activities ${ }^{9}$. Piperazines are found to be antimicrobial agents ${ }^{10-11}$. Structure activity relationship studies of quinazolinone ring system revealed in various literatures ${ }^{12-14}$ suggest position 2,6 and 8 are very much important for structure activity studies and position 3 should be attached to different heterocyclic rings for better chemotherapeutic activity. 
Keeping in view the potential biological activities of quinazolin-4-ones and piperazines, it was perceived that if both the heterocyclic moieties are synergized in a single nucleus, the new compounds obtained were likely to possess significant antimicrobial activities. In this quest, novel 2-(1H-substituted-piperazin-1-yl)- $N$-(4-oxo-substituted-quinazolin-3(4H)-yl)acetamides 8-30 were synthesized by reacting different piperazines with 2-chloro- $N$-(4-oxosubstituted-quinazolin-3(4H)-yl)-acetamides and subjected to antimicrobial activity.

In Scheme-1 $o$-aminobenzoic acid was brominated at $15^{\circ} \mathrm{C}$ in presence of glacial acetic acid to form 2-amino-3,5-dibromobenzoic acid 1. Different anthranilic acids on treatment with benzoyl chloride/acid anhydrides yields substituted-4H-3,1-benzoxazin-4-one 2,3ab, which on condensation with hydrazine hydrate gives 3-amino-substituted-quinazolin-4(3H)one 4,5ab. These 3-amino-quinazolin-4(3H)-ones 4,5ab were reacted with chloroacetyl chloride in toluene to form 2-chloro- $N$-(4-oxo-substituted-quinazolin-3(4H)-yl)-acetamides $\mathbf{6 , 7} \mathbf{a b}$, which were later treated with piperazine derivatives in presence of anhydrous potassium carbonate to yield 2-(1H-substituted-piperazin-1-yl)- $N$-(4-oxo-substitutedquinazolin-3(4H)-yl)-acetamides 8-30. The infrared spectra of the 3 -amino-quinazolin$4(3 H)$-ones 4,5ab showed characteristic absorption bands at $3200-3300 \mathrm{~cm}^{-1}$, was attributed to $\mathrm{NH}_{2}$, which were disappeared by the formation of 2-chloro- $N$-(4-oxo-substitutedquinazolin-3(4H)-yl)-acetamides 6,7ab. Similarly the ${ }^{1}$ HNMR spectra of 2-chloro- $N$-(4-oxosubstituted-quinazolin-3(4H)-yl)-acetamides 6,7ab showed characteristic diastereotopic two doublets at $\delta$ 4.1-4.4 due to $\mathrm{C}=\mathrm{OCH}_{2} \mathrm{Cl}$ protons. Presence of signals at $\delta 2.2-2.4$ due to piperazine protons established that all the 2-chloro- $N$-(4-oxo-substituted-quinazolin-3(4H)yl)-acetamides had converted into 2-(1H-substituted-piperazin-1-yl)- $N$-(4-oxo-substitutedquinazolin-3(4H)-yl)-acetamides 8-30. The ${ }^{1} \mathrm{HNMR}$, mass spectra, IR and elemental analysis supported the structure of title compounds. The synthesis of compounds $\mathbf{8 - 3 0}$ is mentioned in Scheme 1 and their physico-chemical data are given in Table 1.

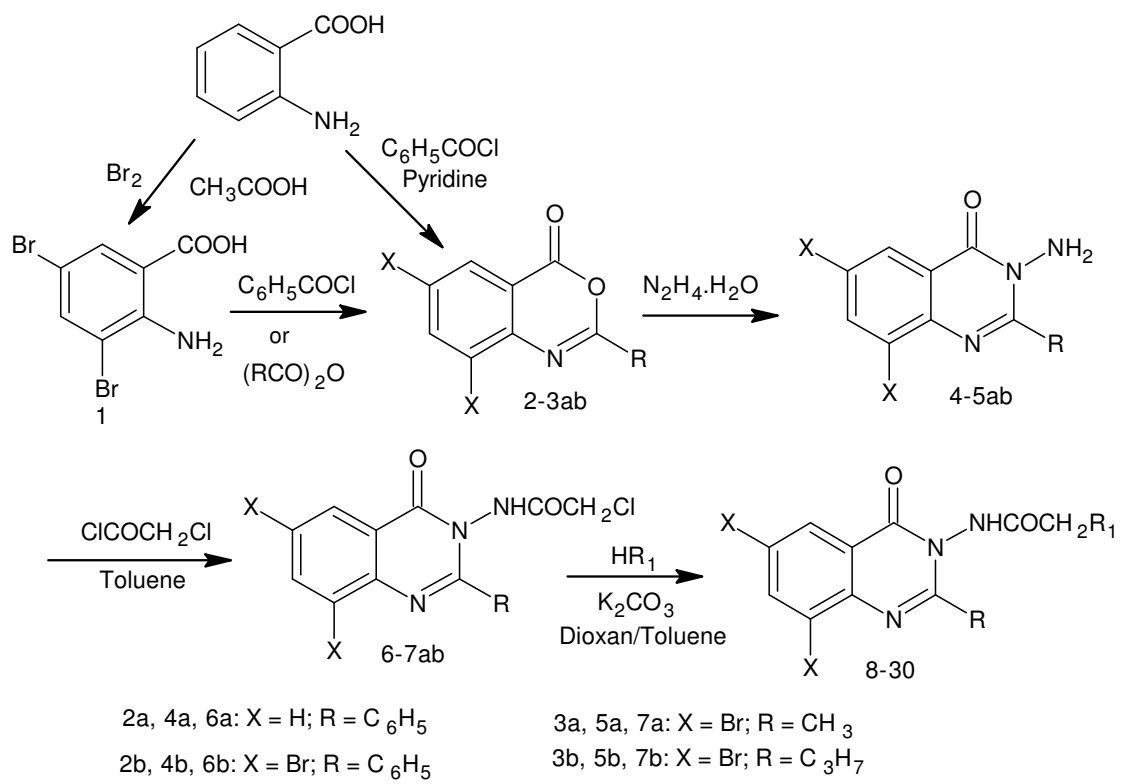

Scheme 1 
Table 1. Physico-chemical data of synthesized compounds (8-30)

\begin{tabular}{|c|c|c|c|c|c|}
\hline Compounds & $\mathrm{X}$ & $\mathrm{R}$ & $\mathrm{R}^{1}$ & Yield, $\%$ & M.P ${ }^{\circ} \mathrm{C}$ \\
\hline 8 & $\mathrm{H}$ & $\mathrm{C}_{6} \mathrm{H}_{5}$ & piperazin-1-yl & 60 & 250 \\
\hline 9 & $\mathrm{H}$ & $\mathrm{C}_{6} \mathrm{H}_{5}$ & 4-methylpiperazin-1-yl & 45 & 210 \\
\hline 10 & $\mathrm{H}$ & $\mathrm{C}_{6} \mathrm{H}_{5}$ & 4-ethylpiperazin-1-yl & 48 & 125 \\
\hline 11 & $\mathrm{H}$ & $\mathrm{C}_{6} \mathrm{H}_{5}$ & 4-phenylpiperazin-1-yl & 50 & 112 \\
\hline 12 & $\mathrm{H}$ & $\mathrm{C}_{6} \mathrm{H}_{5}$ & 4-(4-fluorophenyl)piperazin-1-yl & 38 & 128 \\
\hline 13 & $\mathrm{H}$ & $\mathrm{C}_{6} \mathrm{H}_{5}$ & 4-(2-fluorophenyl)piperazin-1-yl & 40 & 200 \\
\hline 14 & $\mathrm{Br}$ & $\mathrm{C}_{6} \mathrm{H}_{5}$ & piperazin-1-yl & 65 & 285 \\
\hline 15 & $\mathrm{Br}$ & $\mathrm{C}_{6} \mathrm{H}_{5}$ & 4-methylpiperazin-1-yl & 55 & 209 \\
\hline 16 & $\mathrm{Br}$ & $\mathrm{C}_{6} \mathrm{H}_{5}$ & 4-ethylpiperazin-1-yl & 50 & 189 \\
\hline 17 & $\mathrm{Br}$ & $\mathrm{C}_{6} \mathrm{H}_{5}$ & 4-phenylpiperazin-1-yl & 50 & 149 \\
\hline 18 & $\mathrm{Br}$ & $\mathrm{C}_{6} \mathrm{H}_{5}$ & 4-(4-fluorophenyl)piperazin-1-yl & 45 & 205 \\
\hline 19 & $\mathrm{Br}$ & $\mathrm{C}_{6} \mathrm{H}_{5}$ & 4-(2-fluorophenyl)piperazin-1-yl & 47 & 125 \\
\hline 20 & $\mathrm{Br}$ & $\mathrm{CH}_{3}$ & piperazin-1-yl & 68 & 250 \\
\hline 21 & $\mathrm{Br}$ & $\mathrm{CH}_{3}$ & 4-methylpiperazin-1-yl & 45 & 230 \\
\hline 22 & $\mathrm{Br}$ & $\mathrm{CH}_{3}$ & 4-ethylpiperazin-1-yl & 54 & 160 \\
\hline 23 & $\mathrm{Br}$ & $\mathrm{CH}_{3}$ & 4-(4-fluorophenyl)piperazin-1-yl & 52 & 191 \\
\hline 24 & $\mathrm{Br}$ & $\mathrm{CH}_{3}$ & 4-(2-fluorophenyl)piperazin-1-yl & 48 & 126 \\
\hline 25 & $\mathrm{Br}$ & $\mathrm{C}_{3} \mathrm{H}_{7}$ & piperazin-1-yl & 69 & 235 \\
\hline 26 & $\mathrm{Br}$ & $\mathrm{C}_{3} \mathrm{H}_{7}$ & 4-methylpiperazin-1-yl & 57 & 160 \\
\hline 27 & $\mathrm{Br}$ & $\mathrm{C}_{3} \mathrm{H}_{7}$ & 4-ethylpiperazin-1-yl & 54 & 120 \\
\hline 28 & $\mathrm{Br}$ & $\mathrm{C}_{3} \mathrm{H}_{7}$ & 4-phenylpiperazin-1-yl & 49 & 227 \\
\hline 29 & $\mathrm{Br}$ & $\mathrm{C}_{3} \mathrm{H}_{7}$ & 4-(4-fluorophenyl)piperazin-1-yl & 55 & 123 \\
\hline 30 & $\mathrm{Br}$ & $\mathrm{C}_{3} \mathrm{H}_{7}$ & 4-(2-fluorophenyl)piperazin-1-yl & 56 & 179 \\
\hline
\end{tabular}

\section{Experimental}

Thin layer chromatography was used to reach the completion of the reaction and purity of the compounds synthesized. Melting points were taken in open glass capillary tubes by using Thermonik melting point apparatus and were uncorrected. IR spectra in $\mathrm{KBr}$ were recorded on a Shimadzu-8400 FTIR spectrophotometer. ${ }^{1} \mathrm{HNMR}$ spectra were recorded on Brucker spectrophotometer $(400 \mathrm{MHz})$ in DMSO- $\mathrm{d}_{6} / \mathrm{CDCl}_{3}$ using TMS as an internal standard (chemical shifts are expressed in $\delta$, ppm), mass spectra were recorded in Shimadzu QP 5050A Mass spectrometer and micro analysis were recorded on Thermo Finnigan FLASH EA 1122 CHNS Analyzer.

\section{Synthesis of substituted-4H-3,1-benzoxazin-4-one (2,3)ab}

These compounds were synthesized by methods reported earlier ${ }^{15-18}$

General procedure for the synthesis of 2, 6, 8-substituted-3-amino-4-oxoquinazolin3(4H)-one $(4,5) a b$

Respective benzoxazin-4-ones $\mathbf{( 2 , 3 ) a b}(0.01 \mathrm{M})$ was refluxed with hydrazine hydrate $(50 \mathrm{~mL})$ for $3 \mathrm{~h}$ with occasional shaking. The reaction mixture was cooled to room temperature. 
The crystals formed were filtered, washed with water and dried. The products thus formed were recrystallized from ethyl acetate and used in the next step.

\section{3-Amino-2-phenyl-quinazolin-4(3H)-one (4a)}

Yield 40\%; mp $172{ }^{\circ} \mathrm{C}$; IR (KBr) v $\left(\mathrm{cm}^{-1}\right)$ : 3307, $3215(\mathrm{~N}-\mathrm{H}), 3062(\mathrm{ArC}-\mathrm{H}), 1662(\mathrm{C}=\mathrm{O})$, 1564, $1471(\mathrm{ArC}=\mathrm{C}), 1338(\mathrm{C}-\mathrm{N}) ;{ }^{1} \mathrm{HNMR}\left(400 \mathrm{MHz}, \mathrm{DMSO}-d_{6}\right): \delta 8.15-8.25(\mathrm{~d}, 1 \mathrm{H}$, $\mathrm{J}=7.0 \mathrm{~Hz}, \operatorname{ArH}), 7.8-8.1(\mathrm{~m}, 3 \mathrm{H}, \operatorname{ArH}), 7.71-7.73(\mathrm{~d}, 1 \mathrm{H}, \mathrm{J}=8.0 \mathrm{~Hz}, \operatorname{ArH}), 7.55-7.6(t, 1 \mathrm{H}$, $\mathrm{J}=5.6 \mathrm{~Hz}, \mathrm{ArH}), 7.45-7.5(\mathrm{~d}, 3 \mathrm{H}, \mathrm{J}=6.73 \mathrm{~Hz}, \operatorname{ArH}), 5.2\left(\mathrm{~s}, 2 \mathrm{H}, \mathrm{NH}_{2}\right)$.

\section{3-Amino-6,8-dibromo-2-phenyl-quinazolin-4(3H)-one (4b)}

Yield 65\%; mp $235^{\circ} \mathrm{C}$; IR (KBr) $\vee\left(\mathrm{cm}^{-1}\right): 3311,3274(\mathrm{~N}-\mathrm{H}), 3082(\mathrm{ArC}-\mathrm{H}), 1670(\mathrm{C}=\mathrm{O})$, $1568(\mathrm{ArC}=\mathrm{C}), 694(\mathrm{C}-\mathrm{Br}) ;{ }^{1} \mathrm{HNMR}\left(400 \mathrm{MHz}, \mathrm{DMSO}-d_{6}\right): \delta 8.4(\mathrm{~s}, 1 \mathrm{H}, \mathrm{ArH}), 8.2-8.3$ (s, 1H, ArH), 7.8-7.9 (d, 2H, J=6.4Hz, ArH), 7.4-7.6 (m, 3H, ArH), 5.72-5.75 (s, 2H, NH N $^{2}$.

\section{3-Amino-6,8-dibromo-2-methyl-quinazolin-4(3H)-one (5a)}

Yield 65\%; mp $225{ }^{\circ} \mathrm{C}$; IR (KBr) v $\left(\mathrm{cm}^{-1}\right): 3305,3247(\mathrm{~N}-\mathrm{H}), 3076(\mathrm{ArC}-\mathrm{H}), 2952,2862$ $\left(\mathrm{CH}_{3}\right), 1664(\mathrm{C}=\mathrm{O}), 1542,1448(\mathrm{ArC}=\mathrm{C}), 694(\mathrm{C}-\mathrm{Br}) ;{ }^{1} \mathrm{HNMR}\left(400 \mathrm{MHz}, \mathrm{CDCl}_{3}\right): \delta 8.4$ (s, $1 \mathrm{H}, \mathrm{ArH}), 8.2-8.3(\mathrm{~s}, 1 \mathrm{H}, \mathrm{ArH}), 5.8-5.9\left(\mathrm{~s}, 2 \mathrm{H}, \mathrm{NH}_{2}\right), 2.6\left(\mathrm{~s}, 3 \mathrm{H}, \mathrm{CH}_{3}\right)$.

\section{3-Amino-6,8-dibromo-2-propyl-quinazolin-4(3H)-one (5b)}

Yield 60\%; mp $182{ }^{\circ} \mathrm{C}$; IR (KBr) v $\left(\mathrm{cm}^{-1}\right): 3313,3211(\mathrm{~N}-\mathrm{H}), 3086(\mathrm{ArC}-\mathrm{H}), 2978,2861$ $\left(\mathrm{CH}_{3}\right), 2817\left(\mathrm{CH}_{2}\right), 1666(\mathrm{C}=\mathrm{O}), 1606,1589(\mathrm{ArC}=\mathrm{C}), 792\left(\mathrm{CH}_{2}\right), 692(\mathrm{C}-\mathrm{Br}) ;{ }^{1} \mathrm{HNMR}$ $\left(400 \mathrm{MHz}, \mathrm{DMSO}-d_{6}\right): \delta 8.34$ (s, $\left.1 \mathrm{H}, \mathrm{ArH}\right), 8.1-8.2(\mathrm{~s}, 1 \mathrm{H}, \mathrm{ArH}), 5.8\left(\mathrm{~s}, 2 \mathrm{H}, \mathrm{NH}_{2}\right), 2.9-3.1$ $\left(\mathrm{m}, 4 \mathrm{H}, \mathrm{CH}_{2}\right), 1.2-1.4\left(t, 3 \mathrm{H}, \mathrm{J}=7.3 \mathrm{~Hz}, \mathrm{CH}_{3}\right)$.

General method for the synthesis of 2-chloro-N-(2, 6, 8-substituted-4-oxoquinazolin-3(4H)-yl)-acetamide (6,7)ab

2, 6, 8-Substituted-3-amino-4-oxo-quinazolin-3(4H)-one (4,5)ab $(0.018 \mathrm{M})$ was dissolved in $50 \mathrm{~mL}$ of dry toluene and cooled to $15^{\circ} \mathrm{C}$. To this chloroacetyl chloride $(2.3 \mathrm{~mL}, 0.020 \mathrm{M})$ was added drop wise with stirring. The temperature was brought slowly to room temperature and then refluxed for $4 \mathrm{~h}$. Excess toluene was distilled off; precipitate obtained was filtered, washed several times with dry benzene, dried and recrystallized from aqueous dioxan. The completion of the reaction was monitored on silica gel $60 \mathrm{~F}_{254}$ precoated TLC plates by using ethyl acetate, petroleum ether and methanol (1:1:0.3) as the eluent and observed in UV light.

\section{2-Chloro-N-(4-oxo-2-phenyl-quinazolin-3(4H)-yl)-acetamide (6a)}

Yield 55\%; mp $148{ }^{\circ} \mathrm{C}$; IR $(\mathrm{KBr}) \vee\left(\mathrm{cm}^{-1}\right): 3205(\mathrm{~N}-\mathrm{H}), 3010(\mathrm{ArC}-\mathrm{H}), 2935\left(\mathrm{CH}_{2}\right), 1690$ $(\mathrm{C}=\mathrm{O}), 1568,1475(\mathrm{ArC}=\mathrm{C}), 1328(\mathrm{C}-\mathrm{N}), 775(\mathrm{C}-\mathrm{Cl})$; ${ }^{1} \mathrm{HNMR}\left(400 \mathrm{MHz}, \mathrm{DMSO}-d_{6}\right)$ : $\delta 11.5$ (s, 1H, Enol), 8.1-8.2 (d, 2H, J=6.7Hz, ArH), 7.89-7.90 (t, 1H, J=7.0Hz, ArH), 7.75$7.77(\mathrm{~d}, 1 \mathrm{H}, \mathrm{J}=7.9 \mathrm{~Hz}, \operatorname{ArH}), 7.48-7.54(\mathrm{~m}, 5 \mathrm{H}, \operatorname{ArH}), 4.08-4.18(\mathrm{dd}, 2 \mathrm{H}, \mathrm{J}=13.6 \mathrm{~Hz}$, diastereotopic- $\left.\mathrm{CH}_{2}\right)$.

\section{2-Chloro-N-(6,8-dibromo-4-oxo-2-phenyl-quinazolin-3(4H)-yl)-acetamide (6b)}

Yield 58\%; mp $238{ }^{\circ} \mathrm{C}$; IR (KBr) $\vee\left(\mathrm{cm}^{-1}\right): 3230(\mathrm{~N}-\mathrm{H}), 3070(\mathrm{ArC}-\mathrm{H}), 2943\left(\mathrm{CH}_{2}\right), 1710$ $(\mathrm{C}=\mathrm{O}), 1542,1488(\mathrm{ArC}=\mathrm{C}), 700(\mathrm{C}-\mathrm{Cl}), 694(\mathrm{C}-\mathrm{Br}) ;{ }^{1} \mathrm{HNMR}\left(400 \mathrm{MHz}, \mathrm{DMSO}-d_{6}\right): \delta$ 11.6-11.8 (s, 1H, Enol), 8.4-8.5 (s, 1H, ArH), 8.2-8.3 (s, 1H, ArH), 7.4-7.7 (m, 5H, ArH), 4.05-4.20 (dd ,2H, J=13.7Hz, diastereotopic- $\left.\mathrm{CH}_{2}\right)$. 


\section{2-Chloro-N-(6,8-dibromo-2-methyl-4-oxo-quinazolin-3(4H)-yl)-acetamide (7a)}

Yield 60\%; mp $188{ }^{\circ} \mathrm{C}$; IR (KBr) $\vee\left(\mathrm{cm}^{-1}\right): 3188(\mathrm{~N}-\mathrm{H}), 3010(\mathrm{ArC}-\mathrm{H}), 2962,2856\left(\mathrm{CH}_{3}\right)$, $1685(\mathrm{C}=\mathrm{O}), 1583,1438(\mathrm{ArC}=\mathrm{C}), 756(\mathrm{C}-\mathrm{Cl}), 663(\mathrm{C}-\mathrm{Br}) ;{ }^{1} \mathrm{HNMR}\left(400 \mathrm{MHz}, \mathrm{CDCl}_{3}\right)$ : $\delta$ 8.35-8.4 (s, 1H, ArH), 8.15-8.2 (s, 1H, ArH), 4.3-4.5 (dd, 2H, J=13.6Hz, diastereotopic$\left.\mathrm{CH}_{2}\right), 2.35-2.4\left(\mathrm{~s}, 3 \mathrm{H}, \mathrm{CH}_{3}\right)$.

\section{2-Chloro-N-(6,8-dibromo-4-oxo-2-propyl-quinazolin-3(4H)-yl)-acetamide (7b)}

Yield 75\%; mp $192{ }^{\circ} \mathrm{C}$; IR $(\mathrm{KBr}) \vee\left(\mathrm{cm}^{-1}\right): 3301(\mathrm{~N}-\mathrm{H}), 3070(\mathrm{ArC}-\mathrm{H}), 2939\left(\mathrm{CH}_{3}\right)$, $1683(\mathrm{C}=\mathrm{O}), 1585,1442(\mathrm{ArC}=\mathrm{C}), 788\left(\mathrm{CH}_{2}\right), 756(\mathrm{C}-\mathrm{Cl}), 696(\mathrm{C}-\mathrm{Br}) ;{ }^{1} \mathrm{HNMR}$ $\left(400 \mathrm{MHz}, \mathrm{CDCl}_{3}\right): \delta 11.5(\mathrm{~b}, 1 \mathrm{H}, \mathrm{Enol}), 8.45(\mathrm{~s}, 1 \mathrm{H}, \mathrm{ArH}), 8.3(\mathrm{~s}, 1 \mathrm{H}, \mathrm{ArH}), 4.3-4.5$ $\left(\mathrm{dd}, 2 \mathrm{H}, \mathrm{J}=13.7 \mathrm{~Hz}\right.$, diastereotopic- $\left.\mathrm{CH}_{2}\right), 2.58-2.88\left(\mathrm{~m}, 4 \mathrm{H}, \mathrm{CH}_{2}\right), 1.1-1.3(\mathrm{t}, 3 \mathrm{H}$, $\mathrm{J}=7.2 \mathrm{~Hz}, \mathrm{CH}_{3}$ ).

General method for the synthesis of 2-(substituted-piperazin-1-yl)-N-(2,6,8substituted-4-oxo-quinazolin-3(4H)-yl)-acetamide (8-13, 20-30)

2-Chloro- $N$-(2, 6, 8-substituted-4-oxoquinazolin-3(4H)-yl)-acetamide (6a,7ab) $(0.006$ M) was dissolved in $50 \mathrm{~mL}$ of dry toluene, to this freshly dried anhydrous potassium carbonate $(0.9 \mathrm{~g}, 0.0065 \mathrm{M})$ and substituted piperazine $(0.0067 \mathrm{M})$ were added and refluxed for 4-5 h. Excess toluene was distilled off; precipitate obtained was washed with petroleum ether, hot water, dried and recrystallized from aqueous dioxan. The completion of the reaction was monitored on silica gel $60 \mathrm{~F}_{254}$ precoated TLC plates by using ethyl acetate, petroleum ether and methanol (1:1:0.3) as the eluent and observed in UV light.

\section{$\mathrm{N}$-(4-Oxo-2-phenyl-quinazolin-3(4H)-yl)-2-piperazin-1-yl-acetamide (8)}

IR $(\mathrm{KBr}) \vee\left(\mathrm{cm}^{-1}\right): 3180(\mathrm{~N}-\mathrm{H}), 3020(\mathrm{ArC}-\mathrm{H}), 2930,2829\left(\mathrm{CH}_{2}\right), 1710(\mathrm{C}=\mathrm{O}), 1568,1440$ $(\mathrm{ArC}=\mathrm{C}) ;{ }^{1} \mathrm{HNMR}\left(400 \mathrm{MHz}, \mathrm{DMSO}-d_{6}\right): \delta 10.9$ (s, $\left.1 \mathrm{H}, \mathrm{Enol}\right), 8.15-8.25$ (d, $1 \mathrm{H}, \mathrm{J}=7.8 \mathrm{~Hz}$, ArH), 7.9-7.95 (t, 1H, J=7.0 Hz, ArH), 7.75-7.8 (d, 1H, J=8.0Hz, ArH), 7.45-8.25 (m, 6H, $\mathrm{ArH}), \quad 2.8-2.9\left(\mathrm{~d}, 1 \mathrm{H}, \mathrm{J}=15.4 \mathrm{~Hz}\right.$, diastereotopic- $\left.\mathrm{CH}_{2}\right), 3.05-3.15(\mathrm{~d}, 1 \mathrm{H}, \mathrm{J}=15.4 \mathrm{~Hz}$, diastereotopic- $\mathrm{CH}_{2}$ ), 2.3 (s, 4H, Piperazine), 2.1 (s, 4H, Piperazine).

$N$-(2-Phenyl-4-oxo-quinazolin-3(4H)-yl)-2-(4-methylpiperazin-1-yl)-acetamide (9)

IR (KBr) v (cm $\left.{ }^{-1}\right): 3141(\mathrm{~N}-\mathrm{H}), 3060(\mathrm{ArC}-\mathrm{H}), 2937,2808\left(\mathrm{CH}_{2}\right), 1712(\mathrm{C}=\mathrm{O}), 1593,1469$ $(\mathrm{ArC}=\mathrm{C}) ;{ }^{1} \mathrm{HNMR}\left(400 \mathrm{MHz}, \mathrm{DMSO}-d_{6}\right): \delta 11$ (s, 1H, Enol), 7.8-8.25 (m, 3H, ArH), 7.72$7.74(\mathrm{~d}, 1 \mathrm{H}, \mathrm{J}=7.9 \mathrm{~Hz}, \operatorname{ArH}), 7.50-7.65(\mathrm{~m}, 5 \mathrm{H}, \operatorname{ArH}), 2.8-2.9(\mathrm{~d}, 1 \mathrm{H}, \mathrm{J}=15.4 \mathrm{~Hz}$, diastereotopic- $\left.\mathrm{CH}_{2}\right), 3.0-3.2\left(\mathrm{~d}, 1 \mathrm{H}, \mathrm{J}=15.4 \mathrm{~Hz}\right.$, diastereotopic- $\left.\mathrm{CH}_{2}\right), 2.2$ (s, 4H, Piperazine), 2.1 (s, 4H, Piperazine), 1.7 (s, 3H, $\left.\mathrm{CH}_{3}\right)$.

N-(2-Phenyl-4-oxo-quinazolin-3(4H)-yl)-2-(4-ethylpiperazin-1-yl)-acetamide (10)

IR $(\mathrm{KBr}) \vee\left(\mathrm{cm}^{-1}\right): 3142(\mathrm{~N}-\mathrm{H}), 3060(\mathrm{ArC}-\mathrm{H}), 2937,2810\left(\mathrm{CH}_{2}\right), 1710(\mathrm{C}=\mathrm{O}), 1593,1469$ $(\mathrm{ArC}=\mathrm{C}) ;{ }^{1} \mathrm{HNMR}\left(400 \mathrm{MHz}, \mathrm{DMSO}-d_{6}\right): \delta 11(\mathrm{~s}, 1 \mathrm{H}, \mathrm{Enol}), 7.5-8.25(\mathrm{~m}, 9 \mathrm{H}, \mathrm{ArH}), 2.8-2.9$ $\left(\mathrm{d}, 1 \mathrm{H}, \mathrm{J}=15.3 \mathrm{~Hz}\right.$, diastereotopic- $\left.\mathrm{CH}_{2}\right), 3.0-3.2\left(\mathrm{~d}, 1 \mathrm{H}, \mathrm{J}=15.3 \mathrm{~Hz}\right.$, diastereotopic- $\left.\mathrm{CH}_{2}\right), 2.2-$ 2.3 (s, 6H, Piperazine $\left.+\mathrm{CH}_{2}\right), 2.1\left(\mathrm{~s}, 4 \mathrm{H}\right.$, Piperazine), $1.9\left(\mathrm{~s}, 3 \mathrm{H}, \mathrm{CH}_{3}\right)$.

$N$-(2-Phenyl-4-oxo-quinazolin-3(4H)-yl)-2-(4-phenylpiperazin-1-yl)-acetamide (11)

IR (KBr) v (cm $\left.{ }^{-1}\right): 3487(\mathrm{~N}-\mathrm{H}), 3020(\mathrm{ArC}-\mathrm{H}), 2930,2829\left(\mathrm{CH}_{2}\right), 1710(\mathrm{C}=\mathrm{O}), 1568$, $1440(\mathrm{ArC}=\mathrm{C}) ;{ }^{1} \mathrm{HNMR}\left(400 \mathrm{MHz}, \mathrm{DMSO}-d_{6}\right): \delta 11$ (s, 1H, Enol), 8.18-8.25 (d, $1 \mathrm{H}$, 
$\mathrm{J}=7.8 \mathrm{~Hz}, \operatorname{ArH}), 7.9-8.0(\mathrm{t}, 2 \mathrm{H}, \mathrm{J}=7.0 \mathrm{~Hz}, \operatorname{ArH}), 7.4-7.8(\mathrm{~m}, 6 \mathrm{H}, \operatorname{ArH}), 6.9-7.3(\mathrm{~m}, 4 \mathrm{H}$, $\mathrm{ArH}), 6.71-6.73(\mathrm{t}, 1 \mathrm{H}, \mathrm{J}=7.1 \mathrm{~Hz}, \mathrm{ArH}), 3.1-3.2\left(\mathrm{~d}, 1 \mathrm{H}, \mathrm{J}=15.5 \mathrm{~Hz}\right.$, diastereotopic- $\left.\mathrm{CH}_{2}\right)$, 2.9-3.0 (d, $1 \mathrm{H}, \mathrm{J}=15.5 \mathrm{~Hz}$, diastereotopic- $\left.\mathrm{CH}_{2}\right), 2.4-2.6(\mathrm{~m}, 4 \mathrm{H}$, Piperazine), 2.2-2.3 (m, $4 \mathrm{H}$, Piperazine).

$N$-(2-Phenyl-4-oxo-quinazolin-3(4H)-yl)-2-[4-(4-fluorophenyl)piperazin-1-yl]acetamide (12)

IR $(\mathrm{KBr}) \vee\left(\mathrm{cm}^{-1}\right): 3489(\mathrm{~N}-\mathrm{H}), 2920,2818\left(\mathrm{CH}_{2}\right), 1683(\mathrm{C}=\mathrm{O}), 1508,1447(\mathrm{ArC}=\mathrm{C})$, $1233(\mathrm{C}-\mathrm{F}) ;{ }^{1} \mathrm{HNMR}\left(400 \mathrm{MHz}, \mathrm{DMSO}-d_{6}\right): \delta 11$ (s, 1H, Enol), 8.15-8.25 (d, $1 \mathrm{H}$, $\mathrm{J}=7.1 \mathrm{~Hz}, \operatorname{ArH}), 7.9-8.0(\mathrm{t}, 1 \mathrm{H}, \mathrm{J}=8.2 \mathrm{~Hz}, \operatorname{ArH}), 7.75-7.8(\mathrm{~d}, 1 \mathrm{H}, \mathrm{J}=8.0 \mathrm{~Hz}, \operatorname{ArH}), 7.4-7.8$ $(\mathrm{m}, 7 \mathrm{H}, \mathrm{ArH}), 6.9-7.1(\mathrm{~m}, 5 \mathrm{H}, \mathrm{ArH}), 3.1-3.2\left(\mathrm{~d}, 1 \mathrm{H}, \mathrm{J}=15.5 \mathrm{~Hz}\right.$, diastereotopic- $\left.\mathrm{CH}_{2}\right)$, 2.9-3.0 (d, $1 \mathrm{H}, \mathrm{J}=15.5 \mathrm{~Hz}$, diastereotopic- $\left.\mathrm{CH}_{2}\right), 2.4-2.6(\mathrm{~m}, 4 \mathrm{H}$, Piperazine), 2.2-2.3 (m, $4 \mathrm{H}$, Piperazine).

$N$-(2-Phenyl-4-oxo-quinazolin-3(4H)-yl)-2-[4-(2-fluorophenyl)piperazin-1-yl]acetamide (13)

IR $(\mathrm{KBr}) \vee\left(\mathrm{cm}^{-1}\right): 3489(\mathrm{~N}-\mathrm{H}), 2920,2818\left(\mathrm{CH}_{2}\right), 1683(\mathrm{C}=\mathrm{O}), 1508,1447(\mathrm{ArC}=\mathrm{C})$, $1233(\mathrm{C}-\mathrm{F}) ;{ }^{1} \mathrm{HNMR}\left(400 \mathrm{MHz}, \mathrm{DMSO}-d_{6}\right): \delta 11(\mathrm{~s}, 1 \mathrm{H}$, Enol), 8.1-8.2 (d, $1 \mathrm{H}$, $\mathrm{J}=7.6 \mathrm{~Hz}, \operatorname{ArH}), 7.88-7.93(\mathrm{t}, 1 \mathrm{H}, \mathrm{J}=7.3 \mathrm{~Hz}, \operatorname{ArH}), 7.7-7.8(\mathrm{~d}, 1 \mathrm{H}, \mathrm{J}=8.0 \mathrm{~Hz}, \operatorname{ArH}), 7.4-$ $7.7(\mathrm{~m}, 7 \mathrm{H}, \mathrm{ArH}), 6.9-7.2(\mathrm{~m}, 4 \mathrm{H}, \mathrm{ArH}), 3.0-3.1(\mathrm{~d}, 1 \mathrm{H}, \mathrm{J}=14.8 \mathrm{~Hz}$, diastereotopic$\left.\mathrm{CH}_{2}\right), 2.8-2.9\left(\mathrm{~d}, 1 \mathrm{H}, \mathrm{J}=14.8 \mathrm{~Hz}\right.$, diastereotopic- $\left.\mathrm{CH}_{2}\right), 2.4-2.6$ (m, 4H, Piperazine), 2.22.3 ( $\mathrm{m}, 4 \mathrm{H}$, Piperazine).

N-(6,8-Dibromo-2-methyl-4-oxo-quinazolin-3(4H)-yl)-2-piperazin-1-yl-acetamide (20)

IR $(\mathrm{KBr}) \vee\left(\mathrm{cm}^{-1}\right): 3282(\mathrm{~N}-\mathrm{H}), 3030(\mathrm{ArC}-\mathrm{H}), 2932,2844\left(\mathrm{CH}_{2}\right), 1728(\mathrm{C}=\mathrm{O}), 1604,1444$ $(\mathrm{ArC}=\mathrm{C}), 684(\mathrm{C}-\mathrm{Br}) ;{ }^{1} \mathrm{HNMR}\left(400 \mathrm{MHz}, \mathrm{DMSO}-d_{6}\right): \delta 10.9(\mathrm{br}, 1 \mathrm{H}, \mathrm{Enol}), 8.4(\mathrm{~s}, 1 \mathrm{H}$, $\mathrm{ArH}), 8.2(\mathrm{~s}, 1 \mathrm{H}, \mathrm{ArH}), 3.6(\mathrm{~s}, 1 \mathrm{H}, \mathrm{NH}), 3.3-3.4\left(\mathrm{~d}, 1 \mathrm{H}, \mathrm{J}=15.7 \mathrm{~Hz}\right.$, diastereotopic- $\left.\mathrm{CH}_{2}\right), 3.1-$ $3.2\left(\mathrm{~d}, 1 \mathrm{H}, \mathrm{J}=15.7 \mathrm{~Hz}\right.$, diastereotopic- $\mathrm{CH}_{2}$ ), 2.8 (br, 4H, Piperazine), 2.6-2.7 (br, 4H, Piperazine), 2.4 (s, 3H, $\mathrm{CH}_{3}$ ).

N-(6,8-Dibromo-2-methyl-4-oxo-quinazolin-3(4H)-yl)-2-(4-methylpiperazin-1-yl)acetamide (21)

IR $(\mathrm{KBr}) \vee\left(\mathrm{cm}^{-1}\right): 3269(\mathrm{~N}-\mathrm{H}), 3030(\mathrm{ArC}-\mathrm{H}), 2968,2917\left(\mathrm{CH}_{2}\right), 1733(\mathrm{C}=\mathrm{O}), 1602,1465$ $(\mathrm{ArC}=\mathrm{C}), 680(\mathrm{C}-\mathrm{Br}) ;{ }^{1} \mathrm{HNMR}\left(400 \mathrm{MHz}, \mathrm{CDCl}_{3}\right): \delta 8.3-8.35(\mathrm{~s}, 1 \mathrm{H}, \mathrm{ArH}), 8.15(\mathrm{~s}, 1 \mathrm{H}$, ArH), 3.3-3.4 (d, 1H, J=15.6Hz, diastereotopic- $\left.\mathrm{CH}_{2}\right), \quad 3.1-3.2 \quad(\mathrm{~d}, \quad 1 \mathrm{H}, \quad \mathrm{J}=15.6 \mathrm{~Hz}$, diastereotopic- $\mathrm{CH}_{2}$ ), 2.6-2.5 (s, 4H, Piperazine), 2.1-2.2 (s, 4H, Piperazine), 1.25 (s, $3 \mathrm{H}$, $\left.\mathrm{CH}_{3}\right), 0.9$ (s, $\left.3 \mathrm{H}, \mathrm{CH}_{3}\right)$.

$N$-(6,8-Dibromo-2-methyl-4-oxo-quinazolin-3(4H)-yl)-2-(4-ethylpiperazin-1-yl)acetamide (22)

IR $(\mathrm{KBr}) \vee\left(\mathrm{cm}^{-1}\right): 3270(\mathrm{~N}-\mathrm{H}), 3072(\mathrm{ArC}-\mathrm{H}), 2964,2818\left(\mathrm{CH}_{2}\right), 1697(\mathrm{C}=\mathrm{O}), 1598$, $1440(\mathrm{ArC}=\mathrm{C}) ;{ }^{1} \mathrm{HNMR}\left(400 \mathrm{MHz}, \mathrm{CDCl}_{3}\right): \delta 8.2-8.3(\mathrm{~s}, 1 \mathrm{H}, \mathrm{ArH}), 8.15(\mathrm{~s}, 1 \mathrm{H}, \mathrm{ArH})$, 3.4-3.6 (d, $1 \mathrm{H}, \mathrm{J}=15.8 \mathrm{~Hz}$, diastereotopic- $\left.\mathrm{CH}_{2}\right), 3.2-3.4(\mathrm{~d}, 1 \mathrm{H}, \mathrm{J}=15.8 \mathrm{~Hz}$, diastereotopic$\mathrm{CH}_{2}$ ), 3.0-3.2 (br, 4H, Piperazine), 2.8 (br, 4H, Piperazine), 2.8 (br, $2 \mathrm{H}, \mathrm{CH}_{2}$ ), 2.7 (s, $3 \mathrm{H}$, $\mathrm{CH}_{3}$ ), 1.1-1.2 (t, $1 \mathrm{H}, \mathrm{J}=6.8 \mathrm{~Hz}, \mathrm{CH}_{3}$ ). 
N-(6,8-Dibromo-2-methyl-4-oxo-quinazolin-3(4H)-yl)-2-[4-(4-fluorophenyl)piperazin-1-yl]-acetamide (23)

IR $(\mathrm{KBr}) \vee\left(\mathrm{cm}^{-1}\right): 3507(\mathrm{~N}-\mathrm{H}), 3068(\mathrm{ArC}-\mathrm{H}), 2929,2820\left(\mathrm{CH}_{2}\right), 1695(\mathrm{C}=\mathrm{O}), 1597,1443$ $(\mathrm{ArC}=\mathrm{C}), 1240(\mathrm{C}-\mathrm{F}), 672(\mathrm{C}-\mathrm{Br}) ;{ }^{1} \mathrm{HNMR}\left(400 \mathrm{MHz}, \mathrm{DMSO}-d_{6}\right): \delta 8.4(\mathrm{~s}, 1 \mathrm{H}, \mathrm{ArH}), 8.15$ (s, 1H, ArH), 6.9-7.1 (m, 4H, ArH), 3.3-3.4 (d, $1 \mathrm{H}, \mathrm{J}=15.2 \mathrm{~Hz}$, diastereotopic- $\left.\mathrm{CH}_{2}\right), 3.1-3.2$ (d, $1 \mathrm{H}, \mathrm{J}=15.3 \mathrm{~Hz}$, diastereotopic- $\mathrm{CH}_{2}$ ), 2.75-2.85 (m, 4H, Piperazine), 2.65-2.75 (m, 4H, Piperazine), 2.45 (s, $\left.3 \mathrm{H}, \mathrm{CH}_{3}\right)$.

$N$-(6,8-Dibromo-2-methyl-4-oxo-quinazolin-3(4H)-yl)-2-[4-(2-fluorophenyl)piperazin-1-yl]-acetamide (24)

IR $(\mathrm{KBr}) \vee\left(\mathrm{cm}^{-1}\right): 3510(\mathrm{~N}-\mathrm{H}), 3030(\mathrm{ArC}-\mathrm{H}), 2929,2820\left(\mathrm{CH}_{2}\right), 1695(\mathrm{C}=\mathrm{O}), 1597,1443$ $(\mathrm{ArC}=\mathrm{C}), 1240(\mathrm{C}-\mathrm{F}), 700(\mathrm{C}-\mathrm{Br}) ;{ }^{1} \mathrm{HNMR}\left(400 \mathrm{MHz}, \mathrm{DMSO}-d_{6}\right): \delta 11$ (br, 1H, Enol), 8.45 $(\mathrm{s}, 1 \mathrm{H}, \operatorname{ArH}), 8.23(\mathrm{~s}, 1 \mathrm{H}, \operatorname{ArH}), 6.9-7.2(\mathrm{~m}, 4 \mathrm{H}, \operatorname{ArH}), 3.4-3.6(\mathrm{~d}, 1 \mathrm{H}, \mathrm{J}=15.5 \mathrm{~Hz}$, diastereotopic- $\left.\mathrm{CH}_{2}\right), 3.2-3.3\left(\mathrm{~d}, 1 \mathrm{H}, \mathrm{J}=15.6 \mathrm{~Hz}\right.$, diastereotopic- $\left.\mathrm{CH}_{2}\right), 2.8-2.9$ (br, $4 \mathrm{H}$, Piperazine), 2.6-2.8 (br, 4H, Piperazine), 2.43 (s, 3H, $\left.\mathrm{CH}_{3}\right)$.

N-(6,8-Dibromo-4-oxo-2-propyl-quinazolin-3(4H)-yl)-2-piperazin-1-yl-acetamide (25)

IR $(\mathrm{KBr}) \vee\left(\mathrm{cm}^{-1}\right): 3244(\mathrm{~N}-\mathrm{H}), 3030(\mathrm{ArC}-\mathrm{H}), 2945,2835\left(\mathrm{CH}_{2}\right), 1731(\mathrm{C}=\mathrm{O}), 1606,1444$ $(\mathrm{ArC}=\mathrm{C}), 675(\mathrm{C}-\mathrm{Br}) ;{ }^{1} \mathrm{HNMR}\left(400 \mathrm{MHz}, \mathrm{DMSO}-d_{6}\right): \delta 11(\mathrm{br}, 1 \mathrm{H}, \mathrm{Enol}), 8.4(\mathrm{~s}, 1 \mathrm{H}, \mathrm{ArH})$, $8.2(\mathrm{~s}, 1 \mathrm{H}, \mathrm{ArH}), 3.6(\mathrm{~s}, 1 \mathrm{H}, \mathrm{NH}), 3.4-3.5\left(\mathrm{~d}, 1 \mathrm{H}, \mathrm{J}=15.8 \mathrm{~Hz}\right.$, diastereotopic- $\left.\mathrm{CH}_{2}\right), 3.1-3.2(\mathrm{~d}$, $1 \mathrm{H}, \mathrm{J}=15.8 \mathrm{~Hz}$, diastereotopic- $\left.\mathrm{CH}_{2}\right), 2.7-2.9\left(\mathrm{~m}, 6 \mathrm{H}\right.$, Piperazine $\left.+\mathrm{CH}_{2}\right), 2.6-2.7(\mathrm{br}, 6 \mathrm{H}$, Piperazine $\left.+\mathrm{CH}_{2}\right), 1.2-1.3\left(\mathrm{t}, 3 \mathrm{H}, \mathrm{J}=7.2 \mathrm{~Hz}, \mathrm{CH}_{3}\right)$.

$N$-(6,8-Dibromo-4-oxo-2-propyl-quinazolin-3(4H)-yl)-2-(4-methylpiperazin-1-yl)acetamide (26)

IR (KBr) v (cm $\left.{ }^{-1}\right): 3298(\mathrm{~N}-\mathrm{H}), 3030(\mathrm{ArC}-\mathrm{H}), 2937,2819\left(\mathrm{CH}_{2}\right), 1720(\mathrm{C}=\mathrm{O}), 1600,1469$ $(\mathrm{ArC}=\mathrm{C}), 684(\mathrm{C}-\mathrm{Br}) ;{ }^{1} \mathrm{HNMR}\left(400 \mathrm{MHz}, \mathrm{DMSO}-d_{6}\right): \delta 10.9(\mathrm{br}, 1 \mathrm{H}, \mathrm{Enol}), 8.4-8.5(\mathrm{~s}, 1 \mathrm{H}$, $\mathrm{ArH}), 8.2(\mathrm{~s}, 1 \mathrm{H}, \mathrm{ArH}), 3.4-3.5\left(\mathrm{~d}, 1 \mathrm{H}, \mathrm{J}=15.6 \mathrm{~Hz}\right.$, diastereotopic- $\left.\mathrm{CH}_{2}\right), 3.1-3.2(\mathrm{~d}, 1 \mathrm{H}, \mathrm{J}=15.5 \mathrm{~Hz}$, diastereotopic- $\mathrm{CH}_{2}$ ), 2.7-2.8 (m, $\left.2 \mathrm{H}, \mathrm{CH}_{2}\right), 2.6-2.7\left(\mathrm{~m}, 2 \mathrm{H}, \mathrm{CH}_{2}\right), 2.4-2.6$ (br, $4 \mathrm{H}$, Piperazine), 2.3-2.4 (br, 4H, Piperazine), 1.2-1.3 (t, $3 \mathrm{H}, \mathrm{J}=7.3 \mathrm{~Hz}, \mathrm{CH}_{3}$ ); $\mathrm{MS} \mathrm{m} / \mathrm{z}$ : 501 (M) ${ }^{+}$; Calcd. (\%) for $\mathrm{C}_{18} \mathrm{H}_{23} \mathrm{Br}_{2} \mathrm{~N}_{5} \mathrm{O}_{2}$ : C, 43.13; H, 4.63; N, 13.97; Found: C, 43.48; H, 4.32; N, 13.73.

$N$-(6,8-Dibromo-4-oxo-2-propyl-quinazolin-3(4H)-yl)-2-(4-ethylpiperazin-1-yl)acetamide (27)

IR (KBr) v ( $\left.\mathrm{cm}^{-1}\right): 3420(\mathrm{~N}-\mathrm{H}), 2922\left(\mathrm{CH}_{2}\right), 1688(\mathrm{C}=\mathrm{O}), 1605,1443(\mathrm{ArC}=\mathrm{C}), 694(\mathrm{C}-\mathrm{Br})$; ${ }^{1} \mathrm{HNMR}\left(400 \mathrm{MHz}, \mathrm{DMSO}-d_{6}\right): \delta 8.4(\mathrm{~s}, 1 \mathrm{H}, \mathrm{ArH}), 8.2(\mathrm{~s}, 1 \mathrm{H}, \mathrm{ArH}), 3.1-3.2(\mathrm{~d}, 2 \mathrm{H}$, $\mathrm{J}=15.7 \mathrm{~Hz}$, diastereotopic- $\left.\mathrm{CH}_{2}\right), 2.7-2.8\left(\mathrm{~m}, 6 \mathrm{H}\right.$, Piperazine $\left.+\mathrm{CH}_{2}\right), 2.6-2.7(\mathrm{~m}, 6 \mathrm{H}$, Piperazine $\left.+\mathrm{CH}_{2}\right), 2.3\left(\mathrm{t}, 3 \mathrm{H}, \mathrm{J}=7.1 \mathrm{~Hz}, \mathrm{CH}_{3}\right), 1.2-1.25\left(\mathrm{t}, 3 \mathrm{H}, \mathrm{J}=7.0 \mathrm{~Hz}, \mathrm{CH}_{3}\right) ; \mathrm{MS} \mathrm{m} / \mathrm{z}: 515$ $(\mathrm{M})^{+}$; Calcd. (\%) for $\mathrm{C}_{19} \mathrm{H}_{25} \mathrm{Br}_{2} \mathrm{~N}_{5} \mathrm{O}_{2}$ : C, 44.29; H, 4.89; N, 13.59; Found: C, 44.37; H, 4.56; $\mathrm{N}, 13.26$.

$N$-(6,8-Dibromo-4-oxo-2-propyl-quinazolin-3(4H)-yl)-2-(4-phenylpiperazin-1-yl)acetamide (28)

IR $(\mathrm{KBr}) \vee\left(\mathrm{cm}^{-1}\right): 3440(\mathrm{~N}-\mathrm{H}), 2921,2830\left(\mathrm{CH}_{2}\right), 1674(\mathrm{C}=\mathrm{O}), 1586,1445(\mathrm{ArC}=\mathrm{C}), 685$ $(\mathrm{C}-\mathrm{Br}) ;{ }^{1} \mathrm{HNMR}\left(400 \mathrm{MHz}, \mathrm{DMSO}-d_{6}\right): \delta 8.4$ (s, $\left.1 \mathrm{H}, \mathrm{ArH}\right), 8.2$ (s, $\left.1 \mathrm{H}, \mathrm{ArH}\right), 6.8-7.4(\mathrm{~m}, 5 \mathrm{H}$, 
ArH), 3.0-3.25 (m, 2H, diastereotopic- $\left.\mathrm{CH}_{2}\right), 2.5-3.0\left(\mathrm{~m}, 12 \mathrm{H}\right.$, Piperazine $\left.+\mathrm{CH}_{2}\right), 1.25(\mathrm{t}$, $\left.3 \mathrm{H}, \mathrm{J}=7.0 \mathrm{~Hz}, \mathrm{CH}_{3}\right)$.

$N$-(6,8-dibromo-4-oxo-2-propyl-quinazolin-3(4H)-yl)-2-[4-(4-fluorophenyl)piperazin-1-yl]-acetamide (29)

IR $(\mathrm{KBr}) \vee\left(\mathrm{cm}^{-1}\right): 3510(\mathrm{~N}-\mathrm{H}), 3045(\mathrm{ArC}-\mathrm{H}), 2935,2820\left(\mathrm{CH}_{2}\right), 1695(\mathrm{C}=\mathrm{O}), 1597$, $1444(\mathrm{ArC}=\mathrm{C}), 1240(\mathrm{C}-\mathrm{F}), 672(\mathrm{C}-\mathrm{Br}) ;{ }^{1} \mathrm{HNMR}\left(400 \mathrm{MHz}, \mathrm{DMSO}-d_{6}\right): \delta 10.9(\mathrm{~s}, 1 \mathrm{H}$, Enol), $8.4(\mathrm{~s}, 1 \mathrm{H}, \mathrm{ArH}), 8.2(\mathrm{~s}, 1 \mathrm{H}, \mathrm{ArH}), 6.9-7.1(\mathrm{~m}, 4 \mathrm{H}, \mathrm{ArH}), 3.4-3.5(\mathrm{~d}, 1 \mathrm{H}$, $\mathrm{J}=15.6 \mathrm{~Hz}$, diastereotopic- $\left.\mathrm{CH}_{2}\right), 3.0-3.2\left(\mathrm{~d}, 1 \mathrm{H}, \mathrm{J}=15.6 \mathrm{~Hz}\right.$, diastereotopic- $\left.\mathrm{CH}_{2}\right), 2.7-$ $2.9\left(\mathrm{~m}, 6 \mathrm{H}\right.$, Piperazine $\left.+\mathrm{CH}_{2}\right), 2.6-2.7\left(\mathrm{~m}, 6 \mathrm{H}\right.$, Piperazine $\left.+\mathrm{CH}_{2}\right), 1.2-1.3(t, 3 \mathrm{H}$, $\left.\mathrm{J}=7.3 \mathrm{~Hz}, \mathrm{CH}_{3}\right)$.

N-(6,8-Dibromo-4-oxo-2-propyl-quinazolin-3(4H)-yl)-2-[4-(2-fluorophenyl)piperazin-1-yl]-acetamide (30)

IR $(\mathrm{KBr}) \vee\left(\mathrm{cm}^{-1}\right): 3510(\mathrm{~N}-\mathrm{H}), 3045(\mathrm{ArC}-\mathrm{H}), 2935,2820\left(\mathrm{CH}_{2}\right), 1695(\mathrm{C}=\mathrm{O}), 1597$, $1444(\mathrm{ArC}=\mathrm{C}), 1240(\mathrm{C}-\mathrm{F}), 672(\mathrm{C}-\mathrm{Br}) ;{ }^{1} \mathrm{HNMR}\left(400 \mathrm{MHz}, \mathrm{DMSO}-d_{6}\right): \delta 10.9(\mathrm{~s}, 1 \mathrm{H}$, Enol), $8.4(\mathrm{~s}, 1 \mathrm{H}, \operatorname{ArH}), 8.2(\mathrm{~s}, 1 \mathrm{H}, \operatorname{ArH}), 6.9-7.2(\mathrm{~m}, 4 \mathrm{H}, \operatorname{ArH}), 3.4-3.5(\mathrm{~d}, 1 \mathrm{H}$, $\mathrm{J}=15.6 \mathrm{~Hz}$, diastereotopic- $\left.\mathrm{CH}_{2}\right), 3.2-3.3\left(\mathrm{~d}, 1 \mathrm{H}, \mathrm{J}=15.6 \mathrm{~Hz}\right.$, diastereotopic- $\left.\mathrm{CH}_{2}\right), 2.8$ $3.0\left(\mathrm{~m}, 6 \mathrm{H}\right.$, Piperazine $\left.+\mathrm{CH}_{2}\right), 2.6-2.7\left(\mathrm{~m}, 6 \mathrm{H}\right.$, Piperazine $\left.+\mathrm{CH}_{2}\right), 1.25(t, 3 \mathrm{H}$, $\left.\mathrm{J}=7.2 \mathrm{~Hz}, \mathrm{CH}_{3}\right)$.

General method for the synthesis of 2-(substituted-imidazol-1-yl)-N-(2,6,8substituted-4-oxo-quinazolin-3(4H)-yl)-acetamide (14-19)

2-Chloro- $N$-(6,8-dibromo-4-oxo-2-phenyl-quinazolin-3(4H)-yl)-acetamide(3b), 0.006 M) was dissolved in $50 \mathrm{~mL}$ of dry dioxan, to this freshly dried anhydrous potassium carbonate $(0.9 \mathrm{~g}, 0.0065 \mathrm{M})$ and different piperazines $(0.0067 \mathrm{M})$ were added and refluxed for $4 \mathrm{~h}$. Excess dioxan was distilled off; precipitate obtained was washed with hot water, dried and recrystallized from aqueous dioxan. The completion of the reaction was monitored on silica gel $60 \mathrm{~F}_{254}$ precoated TLC plates by using ethyl acetate, petroleum ether and methanol (1:1:0.3) as the eluent and observed in UV light.

$N$-(6,8-Dibromo-4-oxo-2-phenyl-quinazolin-3(4H)-yl)-2-piperazin-1-yl-acetamide (14)

IR $(\mathrm{KBr}) \vee\left(\mathrm{cm}^{-1}\right): 3480(\mathrm{~N}-\mathrm{H}), 3062(\mathrm{ArC}-\mathrm{H}), 2943,2819\left(\mathrm{CH}_{2}\right), 1697(\mathrm{C}=\mathrm{O}), 1589,1448$ $(\mathrm{ArC}=\mathrm{C}), 700(\mathrm{C}-\mathrm{Br}) ;{ }^{1} \mathrm{HNMR}\left(400 \mathrm{MHz}, \mathrm{DMSO}-d_{6}\right): \delta 11$ (s, 1H, Enol), $8.5(\mathrm{~s}, 1 \mathrm{H}, \mathrm{ArH})$, $8.2(\mathrm{~s}, 1 \mathrm{H}, \mathrm{ArH}), 7.4-7.7(\mathrm{~m}, 5 \mathrm{H}, \mathrm{ArH}), 3.0-3.1\left(\mathrm{~d}, 1 \mathrm{H}, \mathrm{J}=15.5 \mathrm{~Hz}\right.$, diastereotopic- $\left.\mathrm{CH}_{2}\right), 2.8-$ 2.9 (d, $1 \mathrm{H}, \mathrm{J}=15.5 \mathrm{~Hz}$, diastereotopic- $\mathrm{CH}_{2}$ ), 2.2-2.3 (br, 4H, Piperazine), 2.0-2.1 (br, 4H, Piperazine); MS m/z: $521(\mathrm{M})^{+}$; Calcd. (\%) for $\mathrm{C}_{20} \mathrm{H}_{19} \mathrm{Br}_{2} \mathrm{~N}_{5} \mathrm{O}_{2}$ : C, 46.09; H, 3.67; N, 13.44; Found: C, 46.35; H, 3.42; N, 13.37 .

N-(6,8-Dibromo-2-phenyl-4-oxo-quinazolin-3(4H)-yl)-2-(4-methylpiperazin-1-yl)acetamide (15)

IR $(\mathrm{KBr}) \vee\left(\mathrm{cm}^{-1}\right)$ : 3294(N-H), $3030(\mathrm{ArC}-\mathrm{H}), 2945,2839\left(\mathrm{CH}_{2}\right), 1737(\mathrm{C}=\mathrm{O}), 1587,1444$ $(\mathrm{ArC}=\mathrm{C}) ;{ }^{1} \mathrm{HNMR}\left(400 \mathrm{MHz}, \mathrm{DMSO}-d_{6}\right)$ : $\delta 11$ (br, $\left.1 \mathrm{H}, \mathrm{Enol}\right), 8.45-8.55(\mathrm{~s}, 1 \mathrm{H}, \mathrm{ArH}), 8.25-$ $8.30(\mathrm{~s}, 1 \mathrm{H}, \mathrm{ArH}), 7.4-7.7(\mathrm{~m}, 5 \mathrm{H}, \mathrm{ArH}), 3.0-3.1\left(\mathrm{~d}, 1 \mathrm{H}, \mathrm{J}=15.5 \mathrm{~Hz}\right.$, diastereotopic- $\left.\mathrm{CH}_{2}\right), 2.8$ $2.9\left(\mathrm{~d}, 1 \mathrm{H}, \mathrm{J}=15.5 \mathrm{~Hz}\right.$, diastereotopic- $\left.\mathrm{CH}_{2}\right), 2.2-2.4$ (br, $4 \mathrm{H}$, Piperazine), $2.14\left(\mathrm{~s}, 3 \mathrm{H}, \mathrm{CH}_{3}\right.$ ), 2.0-2.1 ( $\mathrm{m}, 4 \mathrm{H}$, Piperazine). 
$N$-(6,8-Dibromo-2-phenyl-4-oxo-quinazolin-3(4H)-yl)-2-(4-ethylpiperazin-1-yl)acetamide (16)

IR $(\mathrm{KBr}) \vee\left(\mathrm{cm}^{-1}\right): 3431(\mathrm{~N}-\mathrm{H}), 3010(\mathrm{ArC}-\mathrm{H}), 2948,2812\left(\mathrm{CH}_{2}\right), 1697(\mathrm{C}=\mathrm{O}), 1593,1444$ $(\mathrm{ArC}=\mathrm{C}) ;{ }^{1} \mathrm{HNMR}\left(400 \mathrm{MHz}, \mathrm{DMSO}-d_{6}\right): \delta 8.45-8.55(\mathrm{~s}, 1 \mathrm{H}, \mathrm{ArH}), 8.25(\mathrm{~s}, 1 \mathrm{H}, \mathrm{ArH}), 7.45-$ $7.7(\mathrm{~m}, 7 \mathrm{H}, \mathrm{ArH}), 3.0-3.1\left(\mathrm{~d}, 1 \mathrm{H}, \mathrm{J}=15.7 \mathrm{~Hz}\right.$, diastereotopic- $\left.\mathrm{CH}_{2}\right), 2.8-2.9(\mathrm{~d}, 1 \mathrm{H}, \mathrm{J}=15.7 \mathrm{~Hz}$, diastereotopic- $\left.\mathrm{CH}_{2}\right), 2.2-2.3\left(\mathrm{~m}, 6 \mathrm{H}\right.$, Piperazine $\left.+\mathrm{CH}_{2}\right), 2.0-2.1(\mathrm{~m}, 4 \mathrm{H}$, Piperazine), 0.9-1.0 (t, 3H, J=7.1Hz, $\mathrm{CH}_{3}$ ); MS m/z: $549(\mathrm{M})^{+}$; Calcd. (\%) for $\mathrm{C}_{22} \mathrm{H}_{23} \mathrm{Br}_{2} \mathrm{~N}_{5} \mathrm{O}_{2}: \mathrm{C}, 48.11 ; \mathrm{H}, 4.22$; N, 12.75; Found: C, 48.45; H, 4.35; N, 12.53 .

\section{$N$-(6,8-Dibromo-2-phenyl-4-oxo-quinazolin-3(4H)-yl)-2-(4-phenylpiperazin-1-yl)- acetamide (17)}

IR $(\mathrm{KBr}) \vee\left(\mathrm{cm}^{-1}\right): 3245(\mathrm{~N}-\mathrm{H}), 2919,2842\left(\mathrm{CH}_{2}\right), 1687(\mathrm{C}=\mathrm{O}), 1585,1456(\mathrm{ArC}=\mathrm{C}), 700$ $(\mathrm{C}-\mathrm{Br}) ;{ }^{1} \mathrm{HNMR}\left(400 \mathrm{MHz}, \mathrm{DMSO}-d_{6}\right): \delta 11(\mathrm{~s}, 1 \mathrm{H}, \mathrm{Enol}), 8.5(\mathrm{~s}, 1 \mathrm{H}, \mathrm{ArH}), 8.3(\mathrm{~s}, 1 \mathrm{H}$, $\operatorname{ArH})$, 7.5-7.7 (m, 5H, ArH), 6.7-7.2 (m, 5H, ArH), 3.2-3.25 (d, 1H, J=15.6Hz, diastereotopic- $\left.\mathrm{CH}_{2}\right), 3.15-3.25\left(\mathrm{~d}, 1 \mathrm{H}, \mathrm{J}=15.6 \mathrm{~Hz}\right.$, diastereotopic- $\left.\mathrm{CH}_{2}\right), 2.93-2.98(\mathrm{br}, 4 \mathrm{H}$, Piperazine), 2.2 (br, 4H, Piperazine).

$N$-(6,8-Dibromo-2-phenyl-4-oxo-quinazolin-3(4H)-yl)-2-[4-(4-fluorophenyl)piperazin-1-yl]-acetamide (18)

IR $(\mathrm{KBr}) \vee\left(\mathrm{cm}^{-1}\right): 3206(\mathrm{~N}-\mathrm{H}), 3068(\mathrm{ArC}-\mathrm{H}), 2948,2825\left(\mathrm{CH}_{2}\right), 1691(\mathrm{C}=\mathrm{O}), 1580,1453$ $(\mathrm{ArC}=\mathrm{C}), 1240(\mathrm{C}-\mathrm{F}) ;{ }^{1} \mathrm{HNMR}\left(400 \mathrm{MHz}, \mathrm{DMSO}-d_{6}\right): \delta 11(\mathrm{~s}, 1 \mathrm{H}, \mathrm{Enol}), 8.5(\mathrm{~s}, 1 \mathrm{H}, \mathrm{ArH})$, $8.3(\mathrm{~s}, 1 \mathrm{H}, \mathrm{ArH}), 7.5-7.7$ (m, 5H, ArH), 6.9-7.1 (m, 5H, ArH), 3.18-3.22 (d, 1H, J=15.6Hz, diastereotopic- $\left.\mathrm{CH}_{2}\right), 2.93-2.97\left(\mathrm{~d}, 1 \mathrm{H}, \mathrm{J}=15.6 \mathrm{~Hz}\right.$, diastereotopic- $\left.\mathrm{CH}_{2}\right), 2.4-2.5(\mathrm{br}, 4 \mathrm{H}$, Piperazine), 2.2-2.3 (br, 4H, Piperazine).

$N$-(6,8-Dibromo-2-phenyl-4-oxo-quinazolin-3(4H)-yl)-2-[4-(2-fluorophenyl)piperazin-1-yl]-acetamide (19)

IR $(\mathrm{KBr}) \vee\left(\mathrm{cm}^{-1}\right): 3206(\mathrm{~N}-\mathrm{H}), 3068(\mathrm{ArC}-\mathrm{H}), 2948,2825\left(\mathrm{CH}_{2}\right), 1691(\mathrm{C}=\mathrm{O}), 1580,1453$ $(\mathrm{ArC}=\mathrm{C}), 1240(\mathrm{C}-\mathrm{F}) ;{ }^{1} \mathrm{HNMR}\left(400 \mathrm{MHz}, \mathrm{DMSO}-d_{6}\right): \delta 11(\mathrm{~s}, 1 \mathrm{H}, \mathrm{Enol}), 8.5(\mathrm{~s}, 1 \mathrm{H}, \mathrm{ArH})$, $8.3(\mathrm{~s}, 1 \mathrm{H}, \mathrm{ArH}), 7.5-7.7$ (m, 5H, ArH), 6.9-7.2 (m, 5H, ArH), 3.1-3.2 (d, 1H, J=15.5Hz, diastereotopic- $\left.\mathrm{CH}_{2}\right), 2.9-3.0\left(\mathrm{~d}, 1 \mathrm{H}, \mathrm{J}=15.5 \mathrm{~Hz}\right.$, diastereotopic- $\left.\mathrm{CH}_{2}\right), 2.5$ (br, 4H, Piperazine), 2.4 (br, 4H, Piperazine).

\section{Antibacterial and antifungal activities}

The antimicrobial activity of title compounds was determined in vitro by using paper disc method ${ }^{19}$ against variety of pathogenic bacteria like Staphylococcus aureus $(S$. aureus) (MTCC 3160), Bacillus subtilis (B. subtilis) (MTCC 441) (gram-positive bacteria), Pseudomonas aeruginosa (P. aeruginosa) (MTCC 424), Klebsiella pneumoniae (K. pneumoniae) (MTCC 3384) (gram-negative bacteria) and fungi Aspergillus awamori (A. awamori) (MTCC 2879), Aspergillus oryzae (A. oryzae) (MTCC 1122), Candida albicans (C. albicans) (MTCC 183), Candida tropicalis (C. tropicalis) (MTCC 461). The agar media for each microorganism were prepared as per by Institute of Microbial Technology, Chandigarh, India. The zone of inhibition in $\mathrm{mm}$ was measured. Concentration of $100 \mu \mathrm{g} / \mathrm{mL}$ of test compounds were prepared by dissolving the compounds in dimethyl sulfoxide (DMSO). The standard drugs used were ciprofloxacin and griseofulvin. Antimicrobial activity of tested compounds towards various bacteria and fungi is recorded in Table 2. 
Table 2. Antimicrobial activity of compounds $\mathbf{8 - 3 0}{ }^{\odot}$

\begin{tabular}{|c|c|c|c|c|c|c|c|}
\hline Compound & $\begin{array}{c}. \\
\text { aureus }\end{array}$ & $\begin{array}{c}B . \\
\text { subtilis }\end{array}$ & $\begin{array}{c}P . \\
\text { aeruginosa }\end{array}$ & $\begin{array}{c}\text {. } \\
\text { pneumoniae }\end{array}$ & $\begin{array}{c}A . \\
\text { awamori }\end{array}$ & $\begin{array}{c}A . \\
\text { oryzae }\end{array}$ & $\begin{array}{c}C . \\
\text { tropicalis }\end{array}$ \\
\hline 8 & - & - & + & - & - & - & - \\
\hline 9 & - & - & + & + & - & - & - \\
\hline 10 & - & - & + & - & - & - & - \\
\hline 11 & - & - & - & - & - & ++ & + \\
\hline 12 & - & - & - & - & - & ++ & + \\
\hline 13 & - & - & + & - & - & ++ & - \\
\hline 14 & - & - & - & - & ++ & ++ & - \\
\hline 15 & - & - & + & - & - & - & - \\
\hline 16 & - & + & - & - & - & ++ & ++ \\
\hline 17 & - & + & - & - & - & ++ & ++ \\
\hline 18 & - & - & ++ & - & - & ++ & ++ \\
\hline 19 & - & - & ++ & - & - & ++ & ++ \\
\hline 20 & - & - & - & - & - & + & - \\
\hline 21 & - & - & + & - & ++ & - & - \\
\hline 22 & - & - & ++ & - & - & ++ & ++ \\
\hline 23 & - & - & - & - & - & ++ & ++ \\
\hline 24 & - & - & - & - & - & + & ++ \\
\hline 25 & ++ & - & ++ & - & - & ++ & ++ \\
\hline 26 & - & - & + & - & - & + & + \\
\hline 27 & - & - & - & - & - & + & + \\
\hline 28 & - & - & ++ & - & - & ++ & ++ \\
\hline 29 & - & + & ++ & + & - & ++ & ++ \\
\hline 30 & - & - & + & - & - & - & - \\
\hline Standard $^{\S}$ & ++++ & ++++ & ++++ & ++++ & ++++ & ++++ & ++++ \\
\hline
\end{tabular}

\section{Conclusion}

Simple and moderate yielding reactions of 2-chloro- $N$-(4-oxo-substituted-quinazolin-3(4H)yl)-acetamides with various substituted piperazines to form novel 2-(1H-substitutedpiperazin-1-yl)- $N$-(4-oxo-substituted-quinazolin-3(4H)-yl)-acetamides $\mathbf{8 - 3 0}$ are reported. The antimicrobial screening results revealed that some of the compounds are moderately active. However, the activities of the tested compounds are much less than those of standard antifungal and antibacterial agents used.

\section{Acknowledgements}

The authors are thankful to Indian Institute of Science, Bangalore, for the spectral analysis. The authors are also thankful to Sri. Premnath Reddy, Chairman, Acharya Institutes and Dr. Divakar Goli, Principal, Acharya and B.M. Reddy College of Pharmacy, Bangalore, for laboratory facilities.

\section{References}

1. Jiang J B, Hesson D P, Dusak B A, Dexter D L, Kang G J and Hamel E, J. Med. Chem. 1990, 33, 1721. 
2. Xia Y, Yang Z N, Hour M J, Kuo S C, Xia P, Bastow K F, Nakanishi Y, Nampoothiri P, Hackl T, Hamel E and Lee K H. Bioorg. Med. Chem. Lett. 2001, 11, 1193.

3. Trivedi P B, Undavia N K, Dave A M, Bhatt K N and Desai N C, Indian J. Chem. 1993, 32B, 497.

4. Gangwal N A, Kothawade U R, Galande A D, Pharande D S and Dhake A S, Indian. J. Het. Chem. 2001, 10, 291.

5. Bartroli J, Turmo E, Alguero M, Boncompte E, Vericat M L, Conte L, Ramis J, Merlos M, Rafanell J G and Forn J, J. Med. Chem. 1998, 41, 1869.

6. Alagarsamy V, Revathi R, Meena S, Ramaseshu K V, Rajasekaran S and De-Clerco E, Indian. J. Pharm. Scien. 2004, 4, 459.

7. Gupta D P, Ahmad S, Ashok K and Shanker K, Indian. J. Chem. 1988, 27B, 1060.

8. Chao Q, Deng L, Shih H, Leoni L M, Genini D, Carson D A and Cottam H B, J. Med. Chem. 1999, 42, 3860.

9. Wright W B , Tomcufcik A S, Chan P S, Marsico J W and Press J B, J. Med. Chem. 1987, 30, 2277.

10. Fiebig H H, Berger D P, Kapping K, Ottenheijm H C J and Zylicz Z, J. Cancer Res. Cli. Onco. 1990, 116, 550.

11. Goudgaon N M, Dhondiba V and Vijayalaxmi A, Indian J. Het. Chem. 2004, 13, 271.

12. Hour M J, Huang L J, Kuo S C, Xia Y, Bastow K, Nakanishi Y, Hamel E and Lee K H, J. Med. Chem. 2000, 43, 4479.

13. Kumar S, Shrivastava A K and Sarkar P C, Indian. J. Het. Chem. 1997, 7, 51.

14. Ghorab M M, Abdel-Hamide S G and El-Hakim A E, Indian. J. Het. Chem. 1995, 5, 115.

15. Girija K, Selvam P, Nagarajan R, De Clercq E and Gopal V, Indian J. Het. Chem. 2005, 14, 255.

16. Selvam P, Girija K, Nagarajan G and De Clerco E, 2005, 67, 484.

17. Kumar A, Singh S, Saxena A K and Shanker K, Indian J. Chem. 1988, 27B, 443.

18. El-Naser Ossman A R and El-Sayed Barakat S, Arzneimittel Forschung. 1994, 44, 915.

19. Mathew V, Keshavayya J and Vaidya V P, Eur. J. Med. Chem. 2006, 41, 1048. 


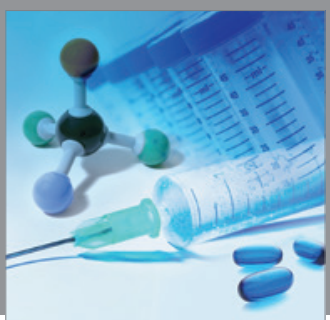

International Journal of

Medicinal Chemistry

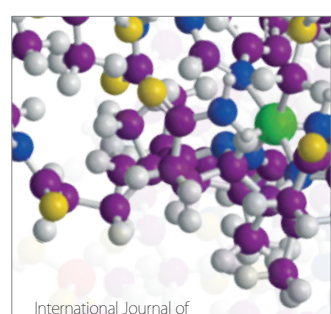

Carbohydrate Chemistry

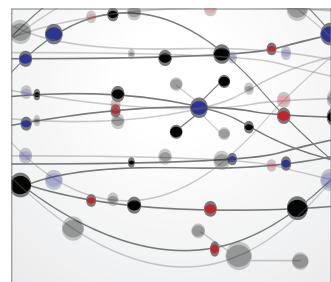

The Scientific World Journal
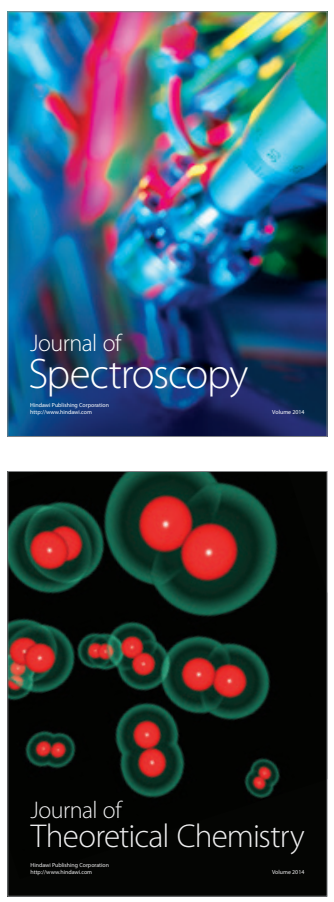
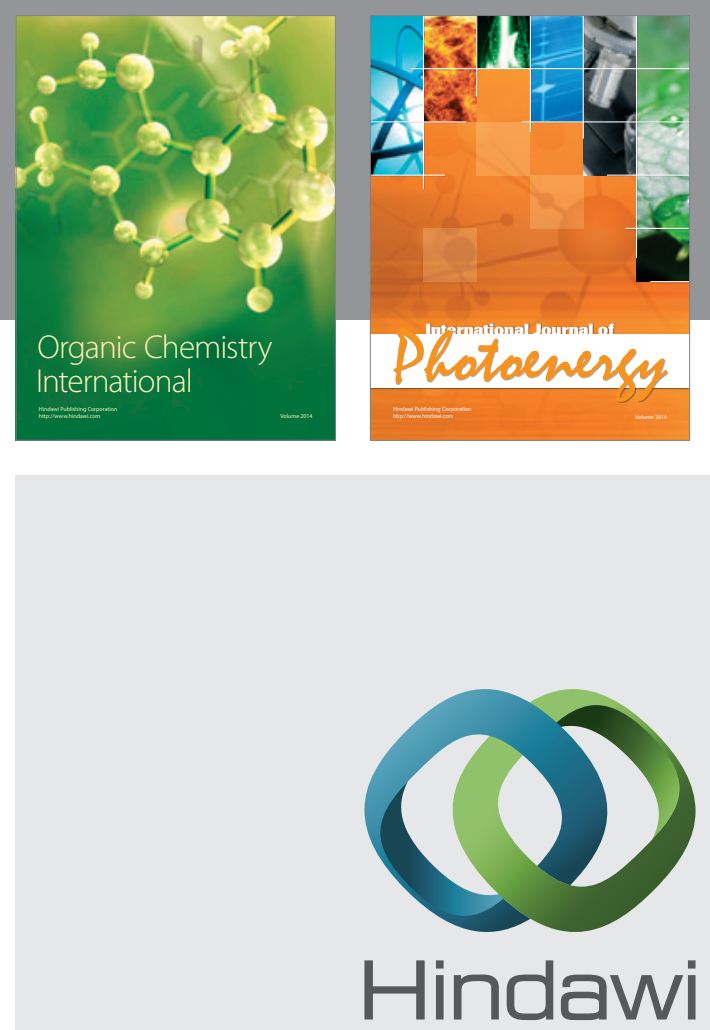

Submit your manuscripts at

http://www.hindawi.com
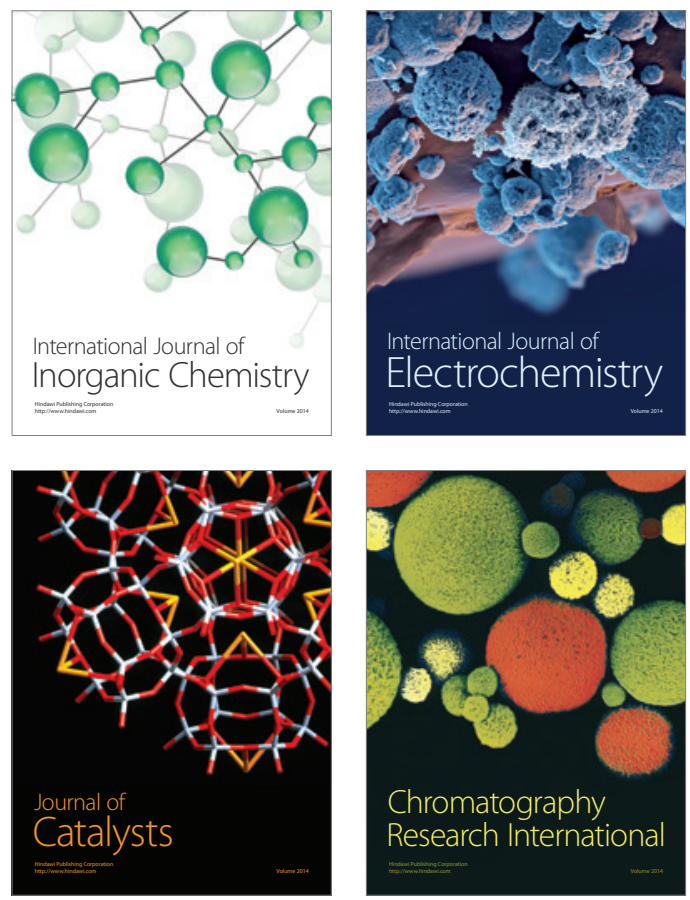
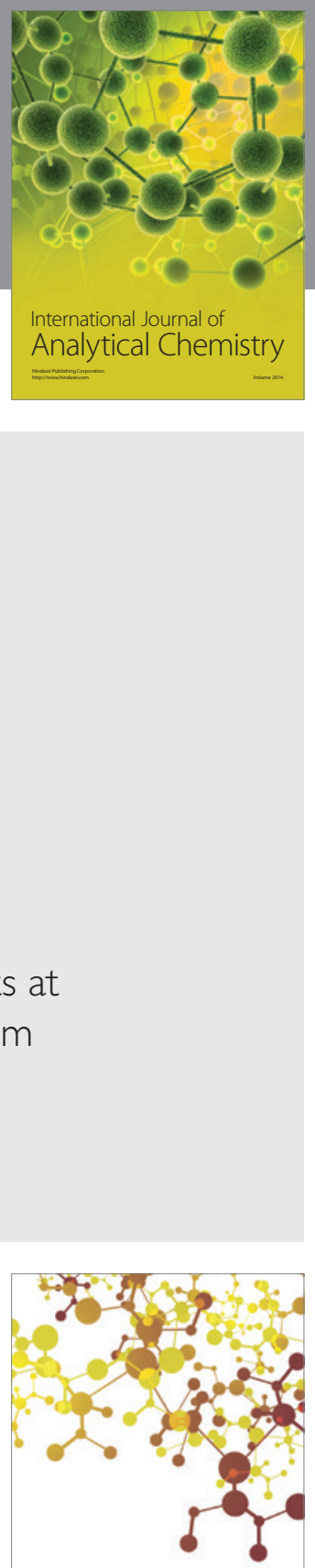

Journal of

Applied Chemistry
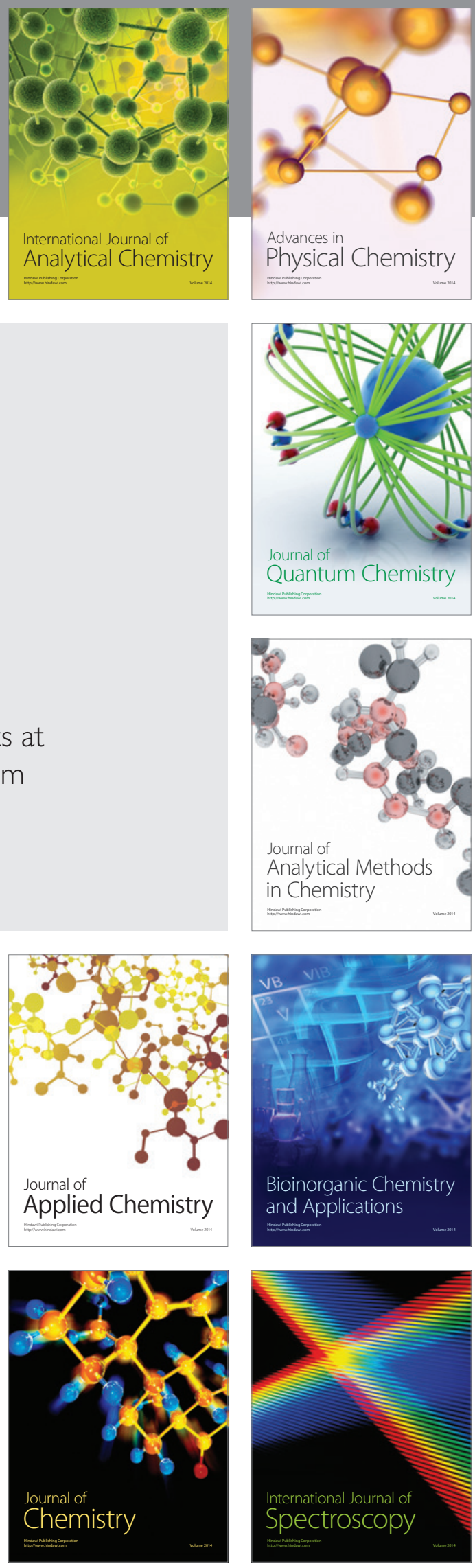